\title{
Wissen schaffen
}

\section{Scientific Inquiry}

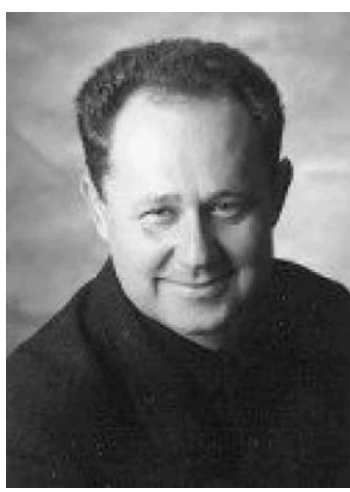

Prof. Dr. Manfred Wildner
Bibliografie

DOI 10.1055/s-0029-1202829

Gesundheitswesen 2009;

71: 119-120

(c) Georg Thieme Verlag KG

Stuttgart · New York

ISSN 0941-3790

Korrespondenzadresse

Prof. Dr. M. Wildner

Bayerisches Landesamt für

Gesundheit und Lebensmittel-

sicherheit

Veterinärstraße 2

85762 Oberschleißheim

manfred.wildner@|gl.bayern.de
„Gute Nachrichten von Einstein - er kommt nicht zurück" - so titelte eine Berliner Zeitung nach dessen Emigration in die USA [1]. Ob dies wirklich eine gute Nachricht war? Im nach hinein war sie es vielleicht, allerdings in anderem Sinne als von den Autoren intendiert: für Einstein. Bereits 1931 war ein Buch „100 Autoren gegen Einstein“ erschienen. „Wenn ich unrecht hätte, wäre einer genug!“, soll seine Antwort darauf gewesen sein $[1,2]$. Der vor 130 Jahren in Ulm geborene Albert Einstein (1879-1955) ist wohl der bekannteste Vertreter der modernen Physik - und damit der Fachdisziplin, welche im 20. Jahrhundert die Funktion einer Leitwissenschaft inne hatte ([3], S. 270). Ob (Evolutions-)Biologie, Neurowissenschaften oder Systemwissenschaften zur Leitwissenschaft des 21. Jahrhunderts avancieren werden, wird sich erst zeigen. Die Erkenntnis, dass sich wissenschaftliche Revolutionen bisweilen erst biologisch mit dem Aussterben ihrer Gegner durchsetzen, ist seit Thomas Kuhn Allgemeingut [4]. Gleiches gilt für die Einsicht, dass wissenschaftliche Karrieren auch zufälligen und risikoreichen „Hasard“-Charakter haben [5]. Einsteins Biografie mit seinen vielfältigen inneren und äußeren Brüchen legt beredtes Zeugnis für Beides ab.

Warum Wissenschaft darüber hinaus thematisieren? Ist sie nicht längst ein Stück selbstverständlicher Normalität? Ist Wissenschaftlichkeit nicht in allen Gesellschaftsbereichen dazu bestimmt, das Verbindende eines disziplinübergreifenden Dialogs darzustellen? Sind antirationale, aufklärungsfeindliche Sonderwege nicht längst passé? Diese Aussagen stimmen und sollen genau deswegen noch einmal betrachtet werden: Wir tun gut daran, den Boden zu kennen, auf dem wir stehen. Der Ursprung des fortschrittsorientierten wissenschaftlichen Programms der Daseinsverbesserung wird dem englischen Philosophen und Staatsmann Francis Bacon (1561-1626) zugeschrieben, pointiert in dem Zitat „[denn auch] Wissen [selbst] ist Macht" [6]. Solche Wissenschaft ist ein Programm der Hoffnung: sie will durch systematisches, der Vernunft verpflichtetes Vorgehen Wissen verfügbar machen, welches uns bei der Daseinsbewältigung hilft. Dass Wissenschaft und Fortschritt miteinander verbunden werden und dass Fortschritt als etwas Positives verstanden wird, ist ein mutiger Gedanke der Neuzeit. Andere Zeiten und andere Kulturen legten und legen ihren Schwerpunkt auf das Be- wahren des Bestehenden, geradezu auf die Abwehr von Veränderung. Kastenwesen, Kult und eine Vielzahl kritikimmunisierter Ideologien liefern Beispiele bis in die Gegenwart. Wissenschaft steht überraschenderweise auch in diesem Sinne vor einem (Selbst-) Anspruch: nämlich Bewahrerin des besten verfügbaren Wissens in der jeweiligen Zeit zu sein.

Francis Bacon könnte zufrieden sein: das 21 Jahrhundert ist durch und durch von den vielfältigen Erfolgen praktischer Wissenschaft geprägt. Vom sekundengenauen Klingeln des Weckers (Astronomie und Atomphysik) über die morgendliche Dusche in Trinkwasserqualität (Hygiene), die Telekommunikations- und Navigationsmöglichkeiten (u.a. Relativitätstheorie), wohlschmeckende Mahlzeiten auf Basis einer ausreichenden Nahrungsmittelproduktion (Lebensmitteltechnologie und Agrarwissenschaften) bis hin zur Medizin mit ihren beeindruckenden Handlungsmöglichkeiten. Im geschichtlichen Verlauf haben sich zudem gesellschaftliche Strukturen herausgebildet - akademische Institutionen, wissenschaftliche Verlage, Kongresse und Belohnungssysteme („science citation impact“) - welche das Programm „Daseinsbewältigung durch wissenschaftlichen Fortschritt“" nachhaltig verankern.

Auch Gesundheit in ihrem Bevölkerungsbezug der Gegenstand von Sozialmedizin, Öffentlicher Gesundheit, Gesundheitssystemforschung, Versorgungsforschung - ist in diesen Prozess einbezogen. Die Baconsche Ausrichtung auf empirische Forschung und prüfende Vernunft hat nachhaltig Einzug gehalten: z.B. als „Evidenz-basierte Medizin“ (EbM), wie die unscharfe Eindeutschung dieses von David Sackett entwickelten Konzeptes lautet [7]. Unscharf wegen der unterschiedlichen Begriffsfelder, welche mit den Bezeichnungen „evident“ im Deutschen und „evidence“ im Englischen verbunden sind. Als Bewegung empirisch belegter Medizin hat sie Einzug gehalten in Sozialgesetzbuch und sozialmedizinische Praxis, von der Bewertung einzelner Publikationen bis zur Erstellung von systematischen Übersichtsarbeiten, Leitlinien und Health Technology Assessments (www.cochrane.de; www.aezq.de; www. dimdi.de). EbM ist auch institutionell präsent, z.B. durch das Institut für Qualität und Wirtschaftlichkeit im Gesundheitswesen (www. iqwig.de). 
Wissenschaft: ein uneingeschränkt gültiges Erfolgsrezept? Dass bei allen Erfolgen der wissenschaftliche Wahrheitsanspruch auch Probleme aufwirft, liegt nicht nur an menschlicher Schwachheit. Die Empörung über Fälle von käuflicher Wissenschaft, von Fälschung, Fabrikation wissenschaftlicher Daten und Plagiarismus ist eher ein Beispiel für die Gültigkeit und Tragfähigkeit des Wahrheitsbezugs in der Wissenschaft als dagegen. Das Problem liegt tiefer, nämlich in der Begründung dieses Wahrheitsbezugs. Hier hat die Wissenschaftstheorie des 20. Jahrhunderts erhebliche Anstrengungen unternommen - sich am eigenen Zopf aus dem Sumpf zu ziehen vermochte sie nicht. Der Wissenschaftstheoretiker Hans Poser hat diese Bemühungen anschaulich dargestellt [3, S. 104-134]. Die klassische Korrespondenztheorie von Wahrheit („Ein wissenschaftlicher Satz ist dann wahr, wenn das als zusammenbestehend ausgesagt wird, was zusammen besteht") scheitert schnell an Aussagen allgemeiner Gesetzmäßigkeiten: kennen wir wirklich jeden Sachverhalt in Vergangenheit, Gegenwart und Zukunft? Die Kohärenztheorie setzt stattdessen auf die Widerspruchsfreiheit von theoretischen Systemen. Dies mag eine Lösung für abstrakte Wissenschaftsbereiche wie die Mathematik sein, jedoch kaum für die anwendungsbezogenen Wissenschaften im Gesundheitswesen. Auch die Konsenstheorie, welche von der intersubjektiven Konkordanz zwischen unvoreingenommenen Wissenschaftlern ausgeht, garantiert nicht Wahrheit. Wissenschaftlicher Fortschritt ist häufig anfänglich eine Minderheitenmeinung und muss sich erst gegen eine verfestigte Mehrheitsmeinung durchsetzen [5]. So gesehen stellen sich die vertrauten Methoden wissenschaftlicher Peer-Reviews und systematischer Übersichtsarbeiten überraschend als angreifbarer Ansatz der Wahrheitsfindung über Expertenkonsens dar.

Man wird Wahrheit im Bereich der Wissenschaft nur als „ein zu approximierendes Ideal [...] ansehen dürfen, ohne doch die Güte der Approximation je feststellen zu können“ [3, S. 106]. Hier hilft auch keine Wahrscheinlichkeitsrechnung weiter: deren Grenzwahrscheinlichkeiten „0“ und „1“ lassen sich nicht mit wahr oder falsch identifizieren. Die Unwahrscheinlichkeit, einen Weitsprung über $8,90 \mathrm{~m}$ selbst in einem weltweiten Studienansatz zu beobachten, sagt nichts über die Tatsächlichkeit eines solchen Sprungs aus. Bob Beamon sprang diese Weite 1968 bei den olympischen Spielen in Mexiko - ein Weltrekord, der den vorangegangenen Rekord um $55 \mathrm{~cm}$ übertraf und 23 Jahre hielt. Dabei können empirische Studien durchaus nützliches Wissen erzeugen: z.B. über die Häufigkeitswahrscheinlichkeit von Sprungleistungen bei jungen Männern.

Kein Platz für die Wahrheit? Alles nur schwankender Boden beliebiger menschlicher Konstruktion? Hier sollte man das Kind nicht mit dem Bade ausschütten: Mögen wissenschaftliche Beobachtungen, Hypothesen und Theorien auch Konstruktionen sein, so sind sie doch in hohem Maß für das Alltagshandeln verlässlich sie bieten zumindest erheblich verbesserte Überlebenschancen.

Hans Poser macht noch eine zweite Feststellung: nämlich die einer Desillusionierung gegenüber der Wissenschaft. Er nennt dafür folgende Gründe: einen Wertkonflikt von Individuum und Wissenschaft, eine unerfüllte Heilserwartung und die unerfüllbare Hoffnung einer Sinnstiftung durch Wissenschaft [3, S. 289]. Der Wertekonflikt begründet sich in dem Spannungsverhältnis zwischen der abendländisch-humanistischen Wertschätzung des Individuums im Kontrast zur „Objektivität“ empirischer Wissenschaftlichkeit, welche der Individualität keinen Raum zu lassen scheint. Die unerfüllte Heilserwartung wird uns vom Geund Missbrauch wissenschaftlich-technischer Möglichkeiten nicht erst seit Hiroshima vor Augen geführt. Dass Handlungswissen allein keinen Sinn stiften kann, wurde schon von Husserl als „Krisis der europäischen Wissenschaften“ thematisiert [8]. Wie problematisch das Machbare werden kann, zeigt die eben nicht „Schöne neue Welt“ genetischen Menschendesigns und letztlich jeder Versuch der „Überflüssigmachung von Menschen als Menschen“ (Aldous Huxley [9] bzw. Hannah Arendt [10]).

Worauf zielen diese Reflexionen? Vor allem auf die Einsicht, dass auch die scheinbar autonome empirische Wissenschaft eine Verankerung in einer anderen Ebene benötigt - und dass sich diese Ebene nicht innerhalb des empirischen Wissenschaftsprogramms festmachen lässt. Letztlich ist das Streben nach empirischem Handlungs- oder Verfügungswissen, dass uns „die Welt verfügbar" machen will, nur eine wissenschaftliche Entwicklungslinie. Eine zweite wichtige Entwicklungslinie gilt dem Orientierungswissen, also der Frage nach (Vor-)Verständnis, Sinn und Deutung. Eine Dritte, ergänzende Entwicklungslinie gilt der Wissenschaftsethik als Regulativ dafür, welche Aspekte wissenschaftlicher Erkenntnis in welcher Form zur Anwendung kommen sollen [11]. Erst diese drei Beine zusammen - Verfügungswissen, Orientierungswissen und Wissenschaftsethik - machen den Schemel „Wissenschaft“ belastbar und tragfähig.

Zurück zu Bevölkerungsgesundheit und Sozialmedizin. „Wissen schaffen" im Dienst der Öffentlichen Gesundheit will und soll in der Tat alles Drei: Handlungsoptionen aufzeigen, Orientierungen geben und all dies ethisch reflektieren. Eine Reduktion auf nur eines dieser Standbeine mag für die Forschungspraxis des oder der Einzelnen zunächst ausreichen, für das Feld insgesamt würde es zu kurz greifen.

Für diese Ausgabe haben sich Autoren, Gutachter und Schriftleitung wieder nach besten Kräften um alle drei Stützen bemüht: Das Heft bietet Originalarbeiten zum Kariesbefall bei Kindern, zu betrieblicher Gesundheitsförderung, zu Patientenpräferenzen in der Rehabilitation und zu Rauchverboten in öffentlichen Räumen. Es orientiert über das Qualitätssicherungsverfahren der GKV in der Rehabilitation und es reflektiert über die Arbeit der Ethikkommissionen bezüglich der klinischen Prüfungen mit Arzneimitteln an Minderjährigen. Ach ja, und auch noch „Gute Nachrichten von Einstein“: Er ist zu einem (historischen) Interview für „Das Gesundheitswesen“ anlässlich seines 130. Geburtstags doch noch einmal kurz zurückgekommen!

\section{Literatur}

1 Hawking SW. Eine kurze Geschichte der Zeit. Die Suche nach der Urkraft des Universums, S. 218 ff. rororo Berlin; 1993

2 Israel H, Ruckhaber E, Weinmann R. 100 Autoren gegen Einstein. Voigtländer, Leipzig; 1931

3 Poser $\mathrm{H}$. Wissenschaftstheorie. Eine philosophische Einführung. Stuttgart, Philipp Reclam jun. GmbH\&Co; 2001

4 Weber $M$. Wissenschaft als Beruf. In: Geistige Arbeit als Beruf. Vier Vorträge vor dem Freistudentischen Bund. (o. O.) 1919, Erster Vortrag. (zitiert in: Sukale M, Hrsg. Max Weber. Schriften zur Wissenschaftslehre. Reclam, Stuttgart; 1991; 240

5 Kuhn T. Die Struktur wissenschaftlicher Revolutionen. Frankfurt a. M., Suhrkamp; 1967

6 Bacon F. Meditationes sacrae, 11. Artikel „De Haeresibus“. In: Essayes. Religious Meditations. Plaies of perswasion and disswasion; 1597

7 Sackett DL, Rosenberg WM, Gray JA et al. Evidence based medicine: what it is and what it isn't. BMJ 1996 Jan 13; 312 (7023): 71-72

8 Husserl E. Die Krisis der europäischen Wissenschaften und die transzendentale Phänomenologie. Hamburg, Meiner; 1996

9 Huxley A. Schöne neue Welt. Frankfurt a. M., Fischer 1953; 2004

10 Arendt, Hannah: Karl Jaspers, Briefwechsel, München-Zürich, Piper $1985 ; 202 \mathrm{ff}$

11 MittelstraßJ. Wissenschaft als Lebensform. Frankfurt a. M., Suhrkamp; 1982; $16 \mathrm{ff}$ 